\title{
Whole genome sequencing reveals identification of new Acinetobacter spp. (maqsudiensis) from local meat samples collected from Dhaka, Bangladesh
}

Muhammad Maqsud Hossain ${ }^{1,2 *}$, Abdus Sadique ${ }^{1}$, Aura Rahman ${ }^{1,2}$, Abdul Khaleque ${ }^{2}$, Abdul Mueed Ibne Momen $^{1,2}$, Mrinmoy Sarker ${ }^{2}$, Tahmina Tabassum ${ }^{1,2}$, Arman Hossain ${ }^{1,2}$, Tasnuva Avzun ${ }^{1}$, Gias U. Ahsan ${ }^{3}$

${ }^{1}$ NSU Genome Research Institute (NGRI), North South University, Baridhara, Basundhara, Dhaka 1229, Bangladesh

${ }^{2}$ Department of Biochemistry and Microbiology, North South University, Dhaka 1229, Bangladesh

${ }^{3}$ Department of Public Health, North South University, Dhaka 1229, Bangladesh

*Correspondence:

Muhammad Maqsud Hossain

Director, NSU Genome Research Institute (NGRI)

North South University, Dhaka 1229

Bangladesh

Phone: 8802-55668200 (ext: 1903)

E-mail: muhammad.maqsud@northsouth.edu; mdmaqsud0@gmail.com 


\section{ABSTRACT}

The advent of next-generation technology has paved way for the discovery of new species which could otherwise be misidentified using conventional cultural and serotyping methods. Acinetobacter is an important nosocomial pathogen frequently isolated from raw and undercooked meat. In this study we announce the draft genome sequence of a newly identified Acinetobacter species cross-reacting with $E$. coli serotype O157:H7. Initially, isolates collected from local meat samples in Dhaka, Bangladesh were cultured in EMB and SMAC for the presumptive identification of O157:H7. However, whole genome and Average Nucleotide Identity (ANI) analyses identified the isolate as a new species within the Acinetobacter genus. The whole genome sequence of this isolate will help to identify potential marker/s of intervention. Further genomic analyses might also shed light onto the virulence properties of this newly identified Acinetobacter species which has been provided the new name of Acinetobacter maqsudiensis.

\section{KEYWORDS:}

Acinetobacter; Bangladesh; next-generation sequencing; average nucleotide identity 


\section{GENOME REPORT}

Different pathogenic bacterial strains isolated from raw and undercooked meat have been associated with many outbreaks of foodborne diseases worldwide (1-5). Major bacterial strains isolated from meat sources include Staphylococcus aureus, Streptococcus spp., Listeria monocytogenes, Bacillus spp., Salmonella spp., Campylobacter spp., Escherichia coli, Yersinia enterocolitica, Acinetobacter spp., Aeromonas spp. and Pseudomonas spp. (2,3,6-9). Acinetobacter species are important nosocomial pathogens $(10,11)$ and infections caused by these organisms include pneumonia, endocarditis, meningitis, skin and wound infections, peritonitis in patients receiving peritoneal dialysis, UTI and bacteremia $(10,11)$. In this investigation we carried out whole genome sequencing of E. coli O157:H7 isolated from meat samples collected from local vendors. However, the characterization of whole genome sequence of one of the suspected E. coli isolates resulted in the identification of a new Acinetobacter spp. We are currently carrying out in depth genomic characterization and other required investigations to confirm this isolate as a new Acinetobacter species.

Initially samples were investigated for the presence of $E$. coli $\mathrm{O} 157: \mathrm{H} 7$ isolated from 50 samples of raw chicken, and meat samples. Meat samples were randomly collected (simple random sampling procedure) from the butchers and shopping centers of different parts of Dhaka City, Bangladesh. All isolates were transferred to NSU Genome Research Institute (NGRI) at North South University, Bangladesh. The specimens were inoculated initially on differential media EMB (HiMedia) and selective media SMAC (Oxoid) for presumptive identification of E. coli O157:H7. Green metallic sheen lines on EMB which had corresponding white colonies on SMAC were selected for latex agglutination test. The isolates were stored in BHI medium containing $15 \%$ glycerol at $-20^{\circ} \mathrm{C}$. Six of these suspected E. coli 0157:H7 isolates were selected for whole genome sequencing. The genomic library was constructed and 300-bp paired-end data was generated using whole-genome sequencing using an Illumina MiSeq platform (Illumina, San Diego, CA, USA) at NGRI. The raw reads were generated $(\sim 15 \times$ coverage $)$ and assembled using SPAdes version 3.11.

Serendipitously one of the six isolates i.e., B51 was confirmed as an Acinetobacter spp. based on its whole genome sequence analysis. This Whole Genome Shotgun project has been deposited at DDBJ/ENA/GenBank under the accession PJRJ00000000. Based on the 16s rRNA phylogeny we initially identified this isolate as Acinetobacter johnsonii, however, analysis of Average Nucleotide Identity (ANI) by GenBank groups provided the information that this isolate could potentially be a new species (Table 1). The ANI calculation suggested that the genome was misidentified and therefore could possibly be a new Acinetobacter species. As this similarity value was lower than the $98.65 \%$ threshold recommended to 
define a new species (12), we suggest the creation of a new species within the genus Acinetobacter named Acinetobacter maqsudiensis, after Muhammad Maqsud Hossain (Director, NGRI) who led the discovery of this new strain.

The findings yet need to be confirmed by further comprehensive analyses using other biochemical parameters and MALDI-TOF-MS spectrum. 
TABLE 1 ANI calculation results of Acinetobacter species whole genome

82.324 (20.8 20.3) 3330008 assembly Acinetobacter gandensis (GCA 001678755.1, ASM167875v1)

81.866 (20.4 21.8) 596228 assembly Acinetobacter _ towneri (GCA 000368785.1, Acin town CIP 107472 V1)

$81.755 \quad 20.2$ 22.0) 1066968 assembly Acinetobacter _towneri (GCA

000688495.1, ASM68849v1)

$81.553 \quad 20.2$ 19.5) 841088 assembly Acinetobacter ${ }_{-}$indicus (GCA

0004요255.1, Acin indi CIP110367 V2)

$82.008 \quad 20.1 \quad 17.4) 595858$ assembly Acinetobacter johnsonii (GCA

000368045.1, Acin john CIP 646 V1)

$81.760 \quad(19.9 \quad 17.7) 596678$ assembly Acinetobacter variabilis (GCA

000369625.1, Acin sp NIPH 2171 V1)

$81.333 \quad$ (19.9 20.1) 1506848 assembly Acinetobacter_indicus (GCA

000830155.1, ASM83015v1)

$81.856 \quad 19.7 \quad 17.6) \quad 1677758$ assembly Acinetobacter johnsonii (GCA

000949655.1, ASM94965v1)

$82.128 \quad(19.5 \quad 18.0) \quad 432348$ assembly Acinetobacter lwoffii (GCA

000248355.2, ASM24835v2)

81.525 (19.4 16.5) 595798 assembly Acinetobacter bohemicus (GCA 000367925.1, Acin sp ANC 3994 V1)

$82.217 \quad$ (19.2 17.7) 596148 assembly Acinetobacter schindleri (GCA

000368625.1, Acin schi CIP107287 V1)

$81.125 \quad$ (19.3 14.7) 774958 assembly Acinetobacter- tandoii (GCA

000400735.1, Acin tand CIP 107469 V1)

$81.089 \quad$ (19.2 14.7) 993398 assembly Acinetobacter_tandoii (GCA

000621065.1, ASM62106v1)

82.276 (18.7 18.1) 596398 assembly Acinetobacter lwoffii (GCA 000369105.1,

Acin lwof CIP_64_10 V1)

$81.263 \quad$ (18.5 18.6) 3397888 assembly Acinetobacter_celticus (GCA

001707755.1 , ASM170775v1)

81.034 (16.2 15.0) 603628 assembly Acinetobacter bouvetii (GCA 000373725.1, ASM37372v1)

$82.479 \quad(15.9 \quad 16.1) 2383068$ assembly Acinetobacter equi (GCA

001307195.1, ASM130719v1)

$80.900 \quad(16.0 \quad 16.2) 3378228$ assembly Acinetobacter_albensis (GCA

900095025.1, IMG-taxon 2671180230 annotated assembly)

82.347 (15.1 12.5) 3383468 assembly Acinetobacter defluvii (GCA 001704615.1, ASM170461v1)

80.692 (15.3 16.6) 1465898 assembly Acinetobacter harbinensis

(GCA 000816495.1, ASM81649v1) 


\section{ACKNOWLEDGEMENTS}

We gratefully acknowledge the Board of Trustee of North South University for funding the project.

\section{REFERENCES}

1. Dehkordi FS, Parsaei P, Saberian S, Moshkelani A, Hajshafiei P, Hoseini RS, et al. Prevalence study of theileria annulata by comparison of four diagnostic techniques in Southwest Iran. Bulg J Vet Med. 2012;15(2):123-30.

2. Momtaz H, Rahimian MD, Dehkordi FS. Identification and characterization of Yersinia enterocolitica isolated from raw chicken meat based on molecular and biological techniques. J Appl Poult Res. 2013;22:137-45.

3. Momtaz H, Dehkordi FS, Rahimi E, Asgarifar A, Momeni M. Virulence genes and antimicrobial resistance profiles of Staphylococcus aureus isolated from chicken meat in Isfahan province, Iran. J Appl Poult Res. 2013;22:913-21.

4. Ghorbani F, Gheisari E, Dehkordi FS. Genotyping of vacA alleles of Helicobacter pylori strains recovered from some Iranian food items. Trop J Pharm Res. 2016;15(8):1631-6.

5. Dehkordi FS, Gandomi H, Basti AA, Misaghi A, Rahimi E. Phenotypic and genotypic characterization of antibiotic resistance of methicillin-resistant Staphylococcus aureus isolated from hospital food. Antimicrob Resist Infect Control. 2017;6(104):1-11.

6. Momtaz H, Dehkordi FS, Taktaz T, Rezvani A, Yarali S. Shiga Toxin-Producing Escherichia coli Isolated from Bovine Mastitic Milk: Serogroups, Virulence Factors, and Antibiotic Resistance Properties. Sci World J. 2012;

7. Rahimi E, Sepehri S, Dehkordi FS, Shaygan S. Prevalence of Yersinia Species in Traditional and Commercial Dairy Products in Isfahan Province, Iran. Jundishapur J Microbiol. 2014;7(4):1-6.

8. Madahi H, Rostami F, Rahimi E, Dehkordi FS. Prevalence of Enterotoxigenic Staphylococcus aureus Isolated From Chicken Nugget in Iran. Jundishapur J Microbiol. 2014;7(8):1-6.

9. Ranjbar R, Masoudimanesh M, Dehkordi FS, Jonaidi-jafari N, Rahimi E. Shiga (Vero)-toxin producing Escherichia coli isolated from the hospital foods; virulence factors, o-serogroups and antimicrobial resistance properties. Antimicrob Resist Infect Control [Internet]. 2017;6(4):1-11. Available from: http://dx.doi.org/10.1186/s13756-016-0163-y

10. Engur D, Cakmak BC, Turkmen MK, Telli M, Eyigor M, Guzunler M. A Milk Pump as a Source for Spreading Acinetobacter baumannii in a Neonatal Intensive Care Unit. Breastfeed Med. 2014;9(10).

11. Gurung M, Nam HM, Tamang MD, Chae MH, Jang GC, Jung SC, et al. Prevalence and antimicrobial susceptibility of Acinetobacter from raw bulk tank milk in Korea. J Dairy Sci. 2013;96(4):1997-2002.

12. Kim M, Oh H, Park S, Chun J. Towards a taxonomic coherence between average nucleotide identity and 16S rRNA gene sequence similarity for species demarcation of prokaryotes. Int J Syst Evol Microbiol. 2014;64:346-51. 